\title{
Time of flight measurements over a radio-link from Uppsala to Bruntingthorpe and their application to testing predictions methods that approximate the ray tracing technique.
}

\author{
M. Pietrella ${ }^{1}$, E.M. Warrington ${ }^{2}$, A.J. Stocker ${ }^{2}$ and C. Bianchi ${ }^{1}$
}

(1) Istituto Nazionale di Geofisica e Vulcanologia, Italy.

(2) Department of Engineering, University of Leicester, LE1 7RH, U.K.

\begin{abstract}
Time of flight measurements (TOF) over the radio link between Uppsala (Tx: $59^{\circ} .9 \mathrm{~N}, 17.6^{\circ} \mathrm{E}$ ) and Bruntingthorpe $\left(\mathrm{Rx}: 52.5^{\circ} \mathrm{N}, 1.1^{\circ} \mathrm{W}\right)$ have been performed every 2 minutes at 6 frequencies $(4.637$ $\mathrm{MHz}, 6.954 \mathrm{MHz}, 8.008 \mathrm{MHz}, 10.391 \mathrm{MHz}, 11.118 \mathrm{MHz}$, and $14.364 \mathrm{MHz}$ ) during the period November 2006 - January 2008. Such measurements have been compared with the TOF provided by three prediction methods that approximate the ray tracing technique: IRI-95, SIRM\&BR_D, and ICEPAC. The root mean square deviation (r.m.s.) between TOF monthly median measurements and TOF monthly median predictions and the differences (DP) between the length of the median and predicted ray path have been calculated. The results, which are presented in terms of r.m.s. and DP for different seasons and different time periods, have indicated that the approximate methods are inadequate and that for more accurate predictions ray tracing techniques should be applied.
\end{abstract}

\section{Introduction}

Accurate predictions of the main parameters characterizing the HF ionospheric channel require a detailed knowledge of the ionospheric conditions that only numerical or analytic ray-tracing techniques can provide. Numerical ray-tracing models (such as Jones and Stephenson, 1975 and Norman et al., 1994) are very accurate but they have a high computational time. Analytic raytracing techniques able to ray trace through horizontal gradients along and in the direction of the ray path, (Norman and Cannon, 1997, 1999), are computationally less intensive than numerical ray tracing, and for this reason their use is particularly advantageous in HF applications as for example in the real-time frequency management of OTH (over-the-horizon) radar systems (Coleman, 1998). In this work three different predictions methods that approximate ray-tracing techniques were tested. The two methods IRI-95 and SIRM\&BR_D are so called because they are based on the monthly median electron density profiles provided by the models IRI-95 (Bilitza, 1990; Bilitza, 2001) and SIRM (Zolesi et al., 1993; Zolesi et al., 1996) in conjunction with the Bradley-Dudeney model (Bradley and Dudeney, 1973), respectively. The third method is called ICEPAC because it is based on the monthly median electron density profiles provided by the software package named ICEPAC (Stewart F.G, undated). A description of these methods will be given in Section 2.

Time of flight measurements (TOF) as well as azimuthal angle of arrival measurements (AOA), on the Uppsala - Bruntingthorpe radio link, have been performed during the period November 2006 January 2008. A description of the analysis conducted to validate the TOF measurements and obtain from these the hourly TOF median measurements associated with on great circle $1 \mathrm{E}$ and $1 \mathrm{~F}$ propagation modes will be given in Section 3.

In order to test the validity of the methods approximating the ray-tracing technique under "quiet" ionospheric conditions, comparisons between the predictions provided by IRI-95, SIRM\&BR_D, ICEPAC and the TOF median measurements for the one hop propagation modes $1 \mathrm{E}$ and $1 \mathrm{~F}$ were carried out for different frequencies and seasons. Some results concerning such comparisons and the discussion of the same are presented in Sections 4 and 5 respectively. 


\section{Description of methods that approximate ray tracing and data analysis}

The International Reference Ionosphere (IRI) (Bilitza, 1990; Bilitza, 2001) is a global empirical model which provides monthly median electron density profiles. Over the years, testing and modifications of IRI has continued in order to improve the model and several versions were produced. In this work the IRI-95 prediction method uses the hourly monthly median electron

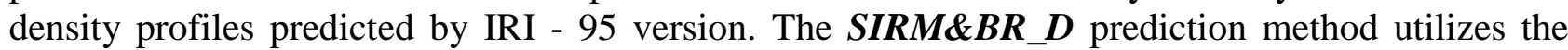
monthly median predictions of the ionospheric characteristics, foF2 and $\mathrm{M}(3000) \mathrm{F} 2$, provided by SIRM (Zolesi et al., 1993; Zolesi et al., 1996) applied in conjunction with the Bradley - Dudeney model (Bradley and Dudeney, 1973) to calculate hourly monthly median electron density profiles. For developing IRI-95 and SIRM\&BR_D prediction methods, the monthly median electron density profiles $N=N(h)$ provided by IRI-95, and SIRM\&BR_D have been used to calculate the vertical plasma frequency profiles $f v=f v(h)$ at the mid-point of the Uppsala (Tx) - Bruntingthorpe $(\mathrm{Rx})$ radio link. As the effect of Earth curvature is important for ground ranges greater than $500 \mathrm{~km}$, the curvature of the Earth and the known ground range $(D=1411 \mathrm{~km})$ of the radio link, have been taken into account for calculating the secant of the angle of incidence $\varphi$ of the ray at the base of the ionosphere as a function of $R, D$, and $h$ ( $R$ being the radius of the Earth) (Davies, 1990). The values of $\sec \varphi=f(R, D, h)$, have been calculated assuming a very simple geometry based on ionospheric reflections taking place from a simple horizontal mirror at the appropriate height $h$ (figure 1). Subsequently, the secant law was applied to calculate the oblique transmission frequencies $f_{o b}=$ $f_{v} \sec \varphi$. The length of the oblique ray path $P=f(R, D$, sec $\varphi)$, its corresponding TOF, and take off angle $\Delta=f(R, \sec \varphi)$ have been calculated from simple mathematical relationships deduced from the geometry sketched in figure 1 . It should be noted that the parameters $f_{o b}, P, T O F, h$, and $\Delta$ (median values) calculated following the procedure described above are strictly related to each other. This means that, if TOF predictions come out reliable after comparing them with TOF median measurements, the other radio propagation path parameters such as $f_{o b}, P, h$, and $\Delta$ should be also considered reliable. In other words, a comparison between TOF median prediction and TOF median measured can be considered suitable for testing indirectly the 'goodness' of prediction of all the other parameters.

The software package ICEPAC (Ionospheric Communications Enhanced Profile Analysis and Circuit) is a full system performance model for HF radio communications circuits (Stewart F.G, undated). Depending on input data it appears in four versions. The version used in this study utilizes the monthly median electron density profiles (Haydon and Lucas., 1968) at the mid-point of the radio link derived from CCIR foF2 and M(3000)F2 coefficients (CCIR, 1966) and, from these, provides for a given oblique transmission frequency and hour, predictions of the main propagation parameters characterizing a given radio link such as elevation angle, TOF, virtual height, etc. 


\section{Data analysis}

Systematic observations of parameters TOF and AOA (azimuthal angle of arrival) were performed from November 2006 to January 2008 over the radio link between Uppsala (Tx: $59.9^{\circ} \mathrm{N}, 17.6^{\circ} \mathrm{E}$ ) and Bruntingthorpe $\left(\mathrm{Rx}: 52.5^{\circ} \mathrm{N}, 1.1^{\circ} \mathrm{W}\right)$ every 2 minutes at 6 frequencies $(4.637 \mathrm{MHz}, 6.954$ $\mathrm{MHz}, 8.008 \mathrm{MHz}, 10.391 \mathrm{MHz}, 11.118 \mathrm{MHz}$, and $14.364 \mathrm{MHz}$ ). The individual observations of TOF have a resolution of $0.1 \mathrm{~ms}$. although the transmitted pulse itself is actually wider than this ( $80 \%$ of the power is contained within $\sim 0.5 \mathrm{~ms}$ ). Since we are looking for the peak, an individual determination has an error of approx. $\pm 0.05 \mathrm{~ms}$ since even after propagation the modes are fairly symmetric. The TOF is calibrated by determining the median TOF of sporadic-E modes over a one year period of measurements and comparing this to the value returned by VOACAP (a free professional HF propagation prediction program, available for download from: http://www.voacap.com/). Some example observations are presented in figures 2-3.

A preliminary analysis was carried out in order to detect the TOF values associated with off great circle propagation. The AOA measurements were used as a filter to determine whether the signal has travelled via a conventional propagation modes, i.e. along the great circle path, or by another mechanism (e.g. deviations due to scattering from irregularities in the auroral oval, Siddle et al. $2004 a, b)$ that results in the signal being displaced significantly from the great circle direction. With this in mind, as the azimuth angle between Uppsala and Bruntingthorpe is approximately $47^{\circ}$, all TOF measurements corresponding to AOA measurements which values differed significantly from $47^{\circ}$, ( i.e. from $47^{\circ} \pm 1.5^{\circ}$ ), were disregarded. In such a way, TOF measurements associated with off great circle propagation modes, interfering signals, or with an array side lobe have been excluded from the analysis and only TOF measurements related to conventional propagation modes selected. Moreover such measurements were "cleaned up" from the calibration offset $(2.7 \pm 0.1 \mathrm{~ms})$ due to the time it takes for the signal to pass through the filters in the transmitter and receiver. Subsequently TOF measurements associated with conventional one hop propagation modes around each hour (hr) were taken into account to extract TOF measurements for $1 \mathrm{E}\left(\mathrm{TOF}_{1 \mathrm{E}, \mathrm{hr}}\right)$ and $1 \mathrm{~F}\left(\mathrm{TOF}_{1 \mathrm{~F}, \mathrm{hr}}\right)$ propagation modes at a given hour. From $\mathrm{TOF}_{1 \mathrm{E} \text {, hr }}$ and $\mathrm{TOF}_{1 \mathrm{~F} \text {, hr }}$ data, TOF median measurements for $1 \mathrm{E}\left(\mathrm{TOF}_{1 \mathrm{E}, \mathrm{hr}}\right.$ med $)$ and $1 \mathrm{~F}\left(\mathrm{TOF}_{1 \mathrm{~F}, \mathrm{hr}}\right.$, med $)$ propagation modes were calculated for each hour, month and frequency.

\section{Results}

A few comparisons between TOF monthly median measurements and TOF monthly median predictions provided by IRI-95, SIRM\&BR_D and ICEPAC are shown in figures 4-6 for different seasons and frequencies for the one hop propagation modes $1 \mathrm{E}$ and $1 \mathrm{~F}$.

Tables 1-2 summarize the performances of the three different methods that approximate ray tracing in terms of r.m.s. difference between TOF monthly median measurements and TOF monthly median predictions over the whole day.

To put better in evidence how important are the TOF prediction errors, the lengths of the median and predicted ray paths, and their differences (DP), have been calculated for all the hours and for all the frequencies. The minimum and maximum DP values are shown for $1 \mathrm{E}$ and $1 \mathrm{~F}$ propagation modes in tables 3-4 respectively. 


\section{Discussion of the results}

Taking into account all the signals at frequencies that arrive at the receiver via $1 \mathrm{E}$ on great circle propagation modes, in winter months (November 2006 to February 2007), equinoctial months (March 2007 and April 2007) and summer months (May 2007 to August 2007) the comparisons between IRI-95 and SIRM\&BR_D do not show significant differences in terms of r.m.s. deviation As expected, both the models are able to predict the TOF of $1 \mathrm{E}$ on great circle propagation modes during daylight hours. The behaviour of ICEPAC is different because it is also able to predict TOF of 1Es propagation modes both during the night time and day time. ICEPAC's performance in general is better than that provided by the IRI-95 and SIRM\&BR_D models as shown in figure 4, but some exceptions are present. In particular the performance of ICEPAC shows a remarkable improvement compared with the performance provided by IRI-95 and SIRM\&BR_D when the sporadic E layer is present as shown in figures 5-6. As can be noted from the results presented in tables 1-4 and figures 4-6, IRI-95, SIRM\&BR_D, and ICEPAC give better predictions when 1E propagation modes are considered.

For $1 \mathrm{E}$ propagation modes, the comparisons among the three predictions methods in table 3 show that the best performance is provided by ICEPAC $\left(\mathrm{DP}_{\max }<20 \mathrm{~km}\right)$, especially during winter months. IRI-95 and SIRM\&BR_D provide the best performance during summer months while does not emerge a significant seasonal difference during winter and equinoctial months $\left(\mathrm{DP}_{\max }>20\right.$ $\mathrm{km})$. Therefore, under these circumstances the prediction methods are not able to approximate accurately the median ray path for $1 \mathrm{E}$ propagation modes given that DP values up to around $30 \mathrm{~km}$ are possible (table 3).

Therefore IRI-95, SIRM\&BR_D, and ICEPAC could be used under ionospheric "quiet" conditions, only to provide a rough estimate of the radio propagation path parameters during daylight hours.

Taking into account all the signals at frequencies that arrive at the receiver via $1 \mathrm{~F}$ on great circle propagation modes the results provided by the three methods are not satisfactory as can be seen in table 2 and 4. A visual review of all the results, reveals that the best performances are given by ICEPAC in January 2007 but only in a few cases (r.m.s. $=0.034 \mathrm{~ms}$ for frequency $=10.391 \mathrm{MHz}$; r.m.s. $=0.025 \mathrm{~ms}$ for frequency $=11.118 \mathrm{MHz}$; r.m.s. $=0.038 \mathrm{~ms}$ for frequency $=14.360 \mathrm{MHz}$ ).

For $1 \mathrm{~F}$ propagation modes, the comparisons in table 4 show that the performance is very bad for each prediction method and season. In fact, except for a very few cases $\mathrm{DP}_{\min }>20 \mathrm{~km}$ and $\mathrm{DP}_{\max }>$ $50 \mathrm{~km}$. DP values of some tens of kilometres (table 4), point out that the predicted ray path is very different from the median ray path. This means that the methods IRI-95, SIRM\&BR_D, and ICEPAC, applied to approximate ray tracing technique, fail completely in providing reliable TOF, $f_{o b}, P, h$, and $\Delta$ predictions under "quiet" ionospheric conditions when $1 \mathrm{~F}$ propagation modes are considered.

\section{Conclusions}

When a radio signal reflects from the $\mathrm{E}$ region, the oblique ray path is affected by the ionosphere for a relatively short time, consequently the model assuming that ionospheric reflection takes place from a simple horizontal mirror is a good approximation. This probably explains why IRI-95 and SIRM\&BR_D can provide under "quiet" ionospheric conditions only a rough estimate of the radio propagation path parameters concerning the $1 \mathrm{E}$ on great circle propagation modes. When the propagation is via the $\mathrm{F}$ region, the ionospheric plasma affects the oblique ray path for a longer time, and consequently the model adopted is not a good approximation. In this case the prediction methods investigated here fail and a precise estimate of the time delay and of all the other radio propagation path parameters related to it cannot be provided also in the case of "quiet" ionosphere. 
This happens because we have tried to characterize the HF ionospheric channel in a very simple way, assuming a geometry based on ionospheric reflections taking place from an horizontal mirror, and using a modelled electron density profile $N=N(h)$ only in the middle point of the radio link, while accurate electron density profiles at all points along the whole path between the transmitter and receiver should be considered for describing with a good accuracy the HF communication link.

At least for the particular radio-link analysed, the results obtained in this work show that, in spite of the relative speed of application of the three prediction methods (no ray tracing is required), it is necessary to adopt a different procedure that is able to include a more appropriate knowledge of the ionospheric conditions. Therefore only a ray tracing technique, either analytical or numerical, could be expected to provide better predictions. The future developments that could improve the predictions are the following:

1. A ray tracing technique with magnetic field, coupled with modelled monthly median electron density profiles in a 2D - 3D ionosphere, could provide large improvements.

2. Elevation angle measurements (EA) collected during the same period (November 2006 to August 2007) and over the same radio link, could be used in conjunction with AOA measurements to conduct a preliminary analysis deeper than the one described in Section 2.

Studies concerning the observed spread in elevation angle versus the observed spread in azimuth angle could be very useful to determine the on great circle propagation modes and to better distinguish both the different propagation modes and the oblique ray paths affected by the presence of tilts.

However, it must be pointed out that these results can not be considered definitive, but only preliminary because they have been obtained just from one particular radio-path. In fact, a systematic study should be carried out taking into account EA, AOA, and TOF measurements over many other radio links located at lower latitudes to draw the final conclusions.

Finally, as regards real time HF applications, as for example in the real-time frequency management of OTH (over-the-horizon) radar systems, new studies could be carried out to develop a 3D monthly median ionospheric model updated, as much as possible, by real-time measurements coming from a network of appropriately located ionosondes. In such a way, a 3D real time ionospheric model, taking into account day-to-day ionospheric variability, coupled with a ray tracing technique could constitute a software package able to provide reliable real time predictions of radio propagation parameters. In such case, real time TOF measurements could be directly compared with TOF predicted to test the "goodness" of the predictions. Obviously, these studies constitute a very different work from that described in this paper. They could be a very interesting work to carry out in future.

\section{Acknowledgements}

The authors would like to thank the unknown referees for their useful comments and suggestions that contributed to improving the paper. 


\section{References}

Bilitza, D. (Ed): The International Reference ionosphere, NSSDC 90-2, World Data Center A, Rockets and Satellites, Greenbelt, USA, 1990.

Bilitza, D. International Reference Ionosphere 2000, Radio Science, Vol 36, No. 2, pp. 261-275, 2001.

Bradley, P.A. and J.R. Dudeney, A simple model of the vertical distribution of electron concentration in the ionosphere. Journal of atmospheric and Terrestrial Physics, Vol 35, 2131-2146, 1973.

CCIR Atlas of ionospheric characteristics, Rept. 340-1, Oslo, ITU, Geneva, Switzerland, 1966.

Coleman, C.J., A ray-tracing formulation and its application to some problems in over-the-horizon radar, Radio Science, Vol 33, No. 4, 1187-1197, 1998.

Davies K. Ionospheric Radio, Peter Peregrinus Ltd. London, 1990

Haydon, G. W., and D.L. Lucas, Predicting ionosphere electron density profiles, Radio Science, Vol. 3, No. 13, 111-119, 1968.

ICEPAC (Ionospheric Communications Enhanced Profile Analysis and Circuit) Stewart F.G, undated. ICEPAC-Technical Manual, available for download at http://www.greghand.com/manuals/icepac_tech_manual.pdf

Jones, R. M., and J.J. Stephenson, A versatile three-dimensional ray tracing computer program, Office of Telecommunications Report 75-76, US Government Printing Office, Washington DC 20402, USA, 1975.

Norman, R. J., J.A. Bennett, P.L. Dyson, and J.A. Nguyen, HIRT: homing-in ray tracing program, research report, School of Phys., La Trobe Univ., Bundoora, Victoria, 1994.

Norman, R. J., and P.S. Cannon, A two-dimensional analytic ray tracing technique accommodating horizontal gradients, Radio Science Vol 32, No. 2, 387-396, 1997

Norman, R. J., and P.S. Cannon, An evaluation of a new two-dimensional analytic ionospheric raytracing technique: Segmented method for analytic ray tracing (SMART). Radio Science, Vol 34, No. 2, 489-499, 1999.

Norman, R. J and P.L. Dyson, HF radar backscatter inversion technique. Radio Science, Vol 41. RS4010, doi:10.1029/2005RS003355, 2006 
Shi. Z., Xu. D. Liu. H. HF detecting radar and communication frequency selection system. Wuhan University Journal of Natural Science. Vol. 5 No.1, 67-69, 2000

Siddle D.R., A.J. Stocker, and E.M. Warrington, Time-of-flight and direction of arrival of HF radio signals received over a path along the mid-latitude trough: observations, Radio Science, Vol 39, RS4008, doi:10.1029/2004RS003049, 2004a.

Siddle D.R., N.Y. Zaalov, A.J. Stocker and E.M. Warrington. Time of flight and direction of arrival of HF radio signals received over a path along the mid-latitude trough: Theoretical considerations, Radio Science, Vol 39, RS4009, doi:10.1029/2004RS003052, 2004b.

Zolesi, B., L.R. Cander, G. De Franceschi, Simplified ionospheric regional model for telecommunication application, Radio Science, Vol. 28, No. 4, 603-612, 1993.

Zolesi, B., L.R. Cander, G. De Franceschi, On the potential applicability of the simplified ionospheric regional model to different mid-latitude areas, Radio Science, Vol. 31, No. 3, 547-552, 1996. 


\section{Figure Captions}

Figure 1. Geometry of reflection from a simple horizontal mirror.

Figure 2. Observations of TOF (on the left) and AOA (on the right) at Uppsala on 27 December 2006 for the transmitting frequencies $4.637 \mathrm{MHz}, 6.954 \mathrm{MHz}, 8.008 \mathrm{MHz}, 10.391 \mathrm{MHz}, 11.118$ $\mathrm{MHz}$, and $14.364 \mathrm{MHz}$; in particular, note the small variations in TOF at around 14.00 U.T. at the frequencies 10.391 and $11.118 \mathrm{MHz}$ indicating a change from $1 \mathrm{~F}$ to $1 \mathrm{E}$ propagation mode.

Figure 3: As for figure 2 but for the day 21 May 2007; in particular, note the change of the signal AOA by several tens of degrees to the north and the corresponding increase in the TOF occurring towards the end of the day at the frequencies 10.391 and $11.118 \mathrm{MHz}$. These AOA and TOF variations show the presence of non -conventional (i.e. off great circle) propagation modes in the ionosphere.

Figure 4. The squares show the TOF provided by IRI-95 prediction method; the circles show the TOF provided by $S I R M \& B R \_D$ prediction method; the triangles show the TOF predictions obtained by ICEPAC. The horizontal line marks the $0 \mathrm{~ms}$ level (no difference observed). For 1E propagation mode, $\boldsymbol{I C E P A C}$ 's performance is slightly better (r.m.s. $=0.038 \mathrm{~ms}$ ) than that provided by $\boldsymbol{I R I - 9 5}$ (r.m.s. $=0.045 \mathrm{~ms}$ ) and by $\boldsymbol{S I R M \& B R \_ D}$ (r.m.s. $\left.=0.045 \mathrm{~ms}\right)$. For $1 \mathrm{~F}$ propagation mode, the performances provided by IRI-95, SIRM\&BR_D and ICEPAC in terms of r.m.s. are equal to $0.325 \mathrm{~ms}, 0.139 \mathrm{~ms}$, and $0.232 \mathrm{~ms}$, respectively.

Figure 5. As for figure 4. For 1E propagation mode, ICEPAC's performance shows a remarkable improvement (r.m.s. $=0.026 \mathrm{~ms}$ ) compared to that provided by $\boldsymbol{I R I - 9 5}$ (r.m.s. $=0.055 \mathrm{~ms}$ ) and by SIRM\&BR_D (r.m.s. $=0.043 \mathrm{~ms})$. For $1 \mathrm{~F}$ propagation mode, the performances provided by $\boldsymbol{I R I}$ 95, SIRM\&BR_D and ICEPAC in terms of r.m.s. are equal to $0.393 \mathrm{~ms}, 0.292 \mathrm{~ms}$, and $0.396 \mathrm{~ms}$, respectively.

Figure 6. As for figure 4. For 1E propagation mode, ICEPAC's performance shows a remarkable improvement (r.m.s. $=0.023 \mathrm{~ms}$ ) compared to that provided by IRI-95 (r.m.s. $=0.046 \mathrm{~ms})$ and by SIRM\&BR_D (r.m.s. $=0.043 \mathrm{~ms})$. For 1F propagation mode, the performances provided by $\boldsymbol{I R I}$ 95, SIRM\&BR_D and ICEPAC in terms of r.m.s. are equal to $0.177 \mathrm{~ms}, 0.161 \mathrm{~ms}$, and $0.234 \mathrm{~ms}$, respectively. 


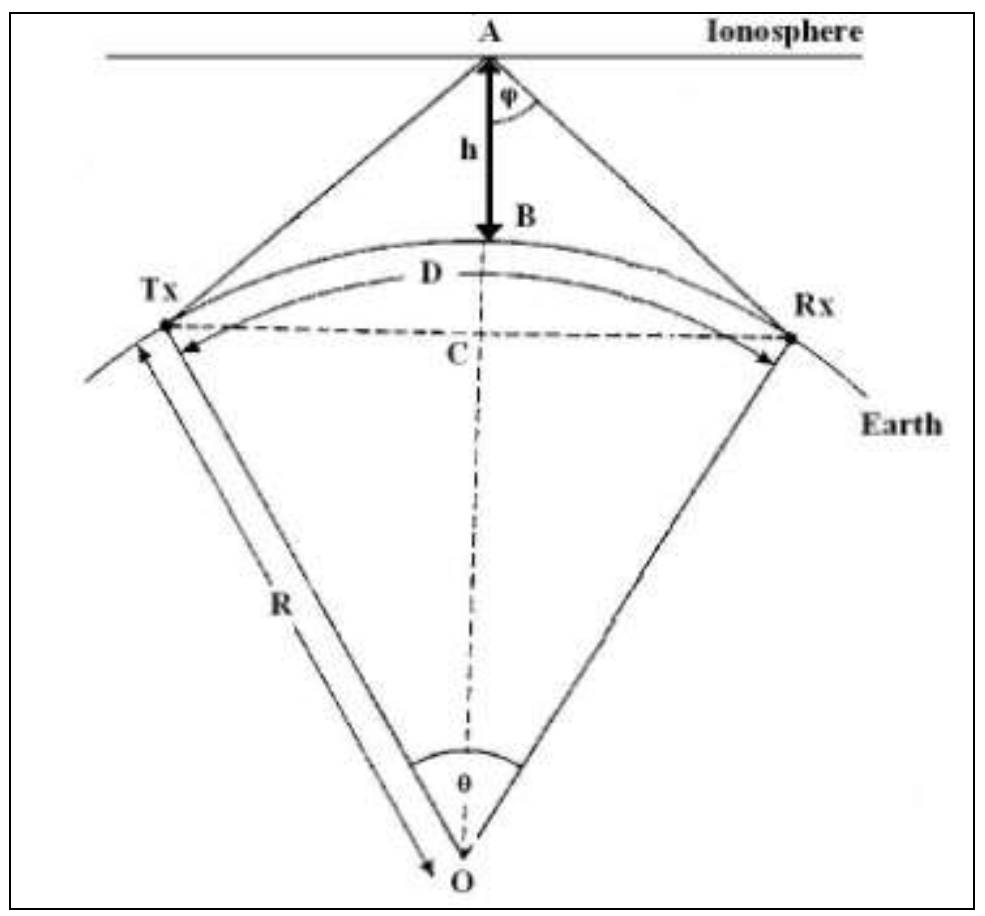

Figure 1 


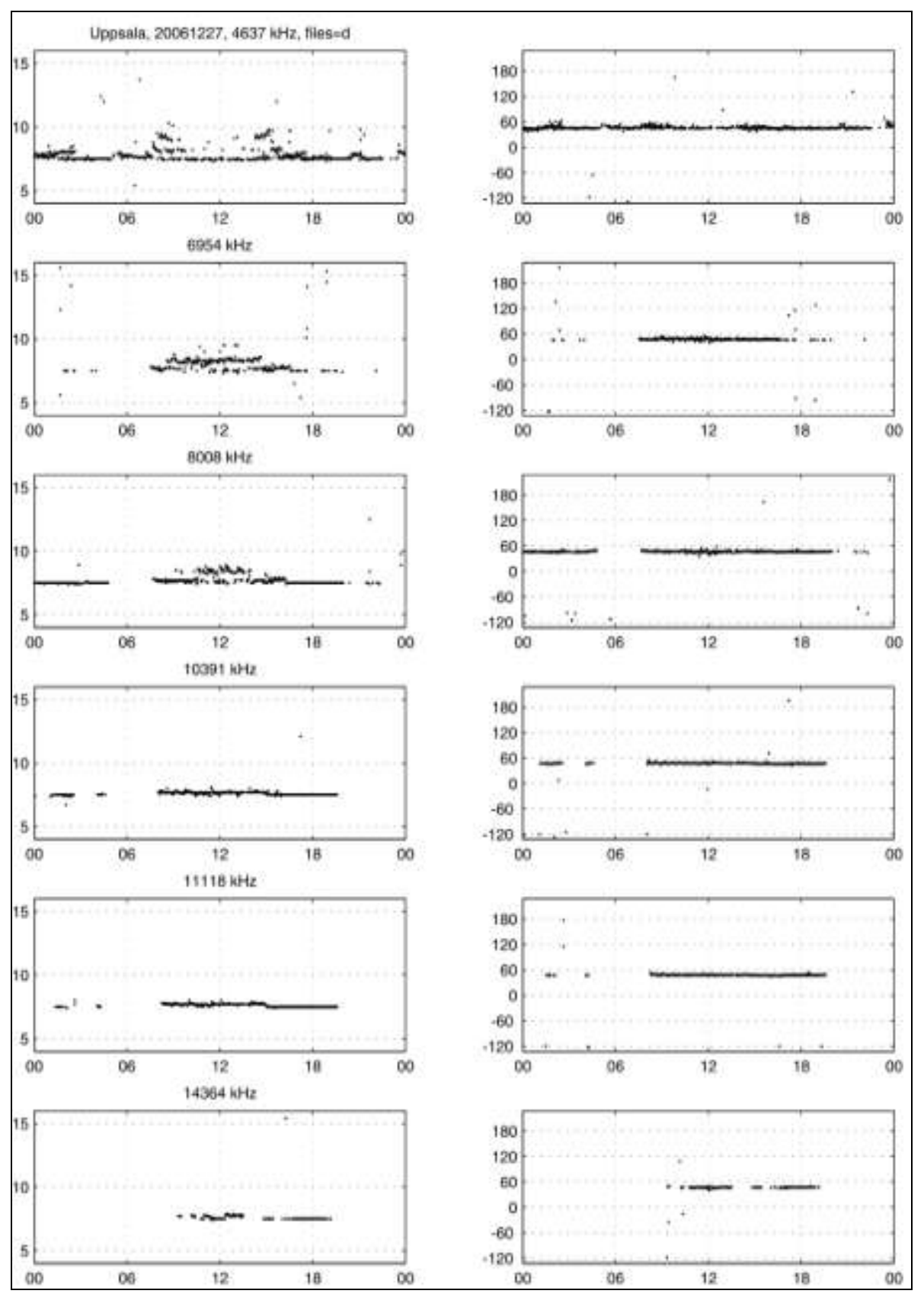

Figure 2 


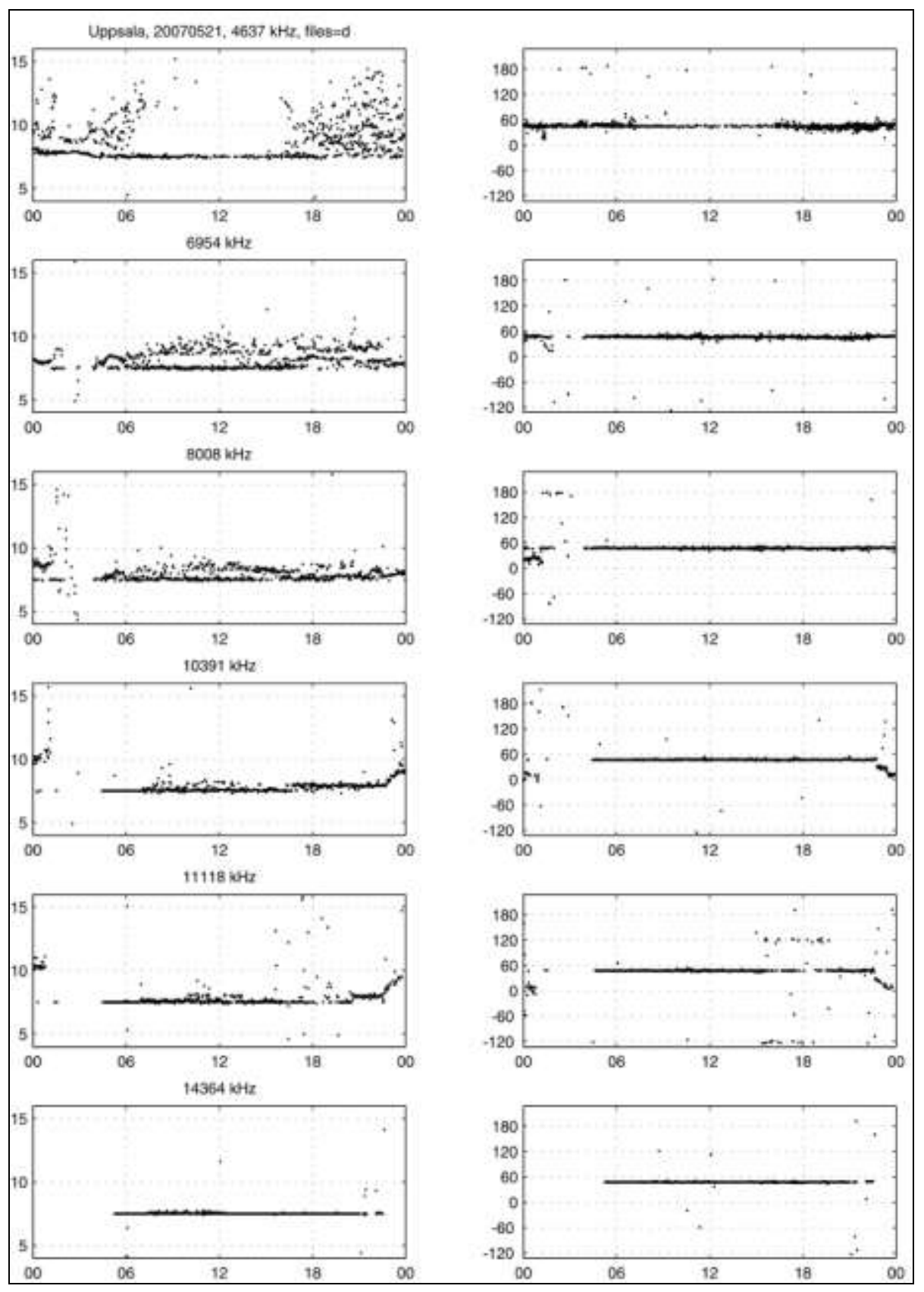

Figure 3 


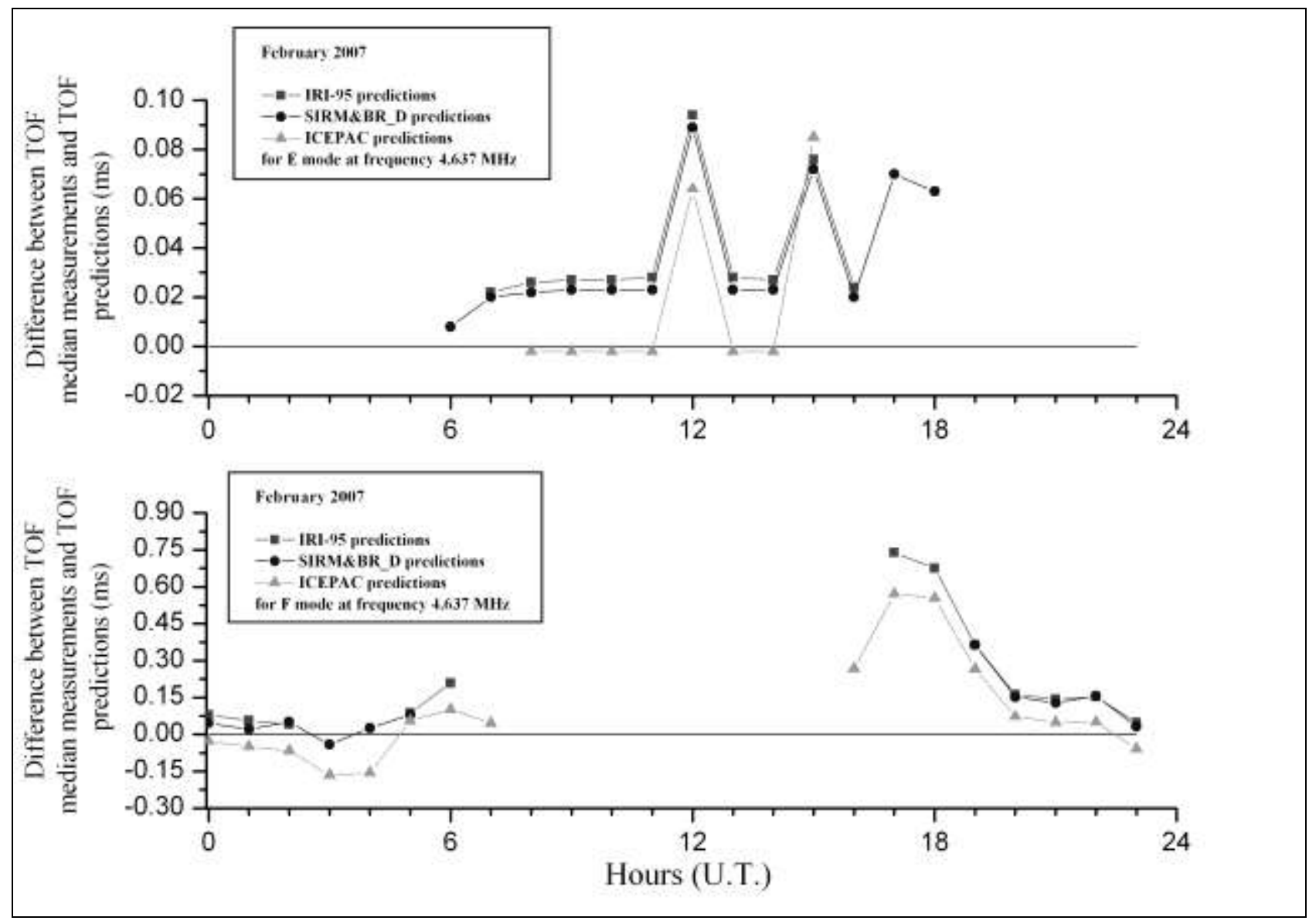

Figure 4 


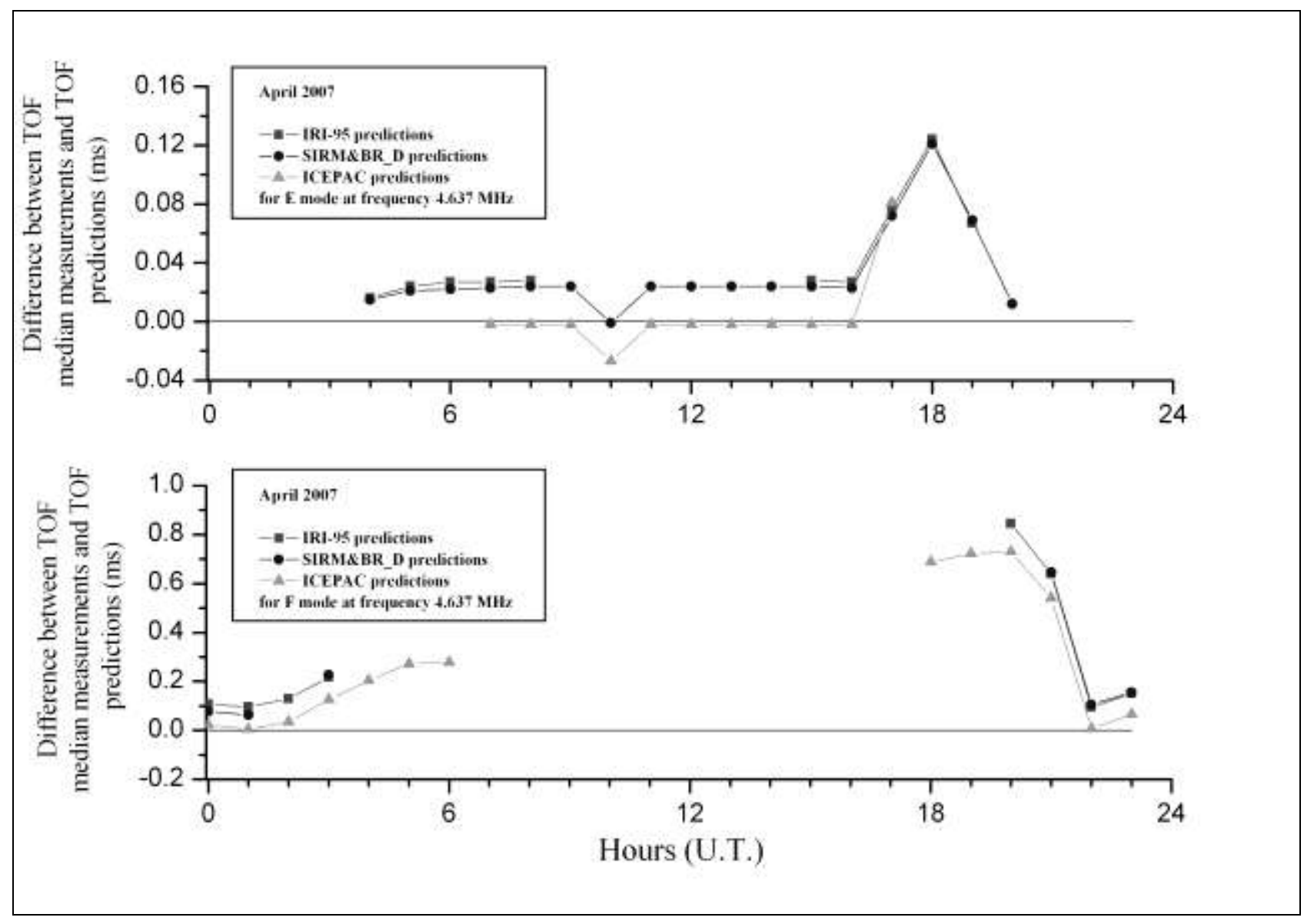

Figure 5 


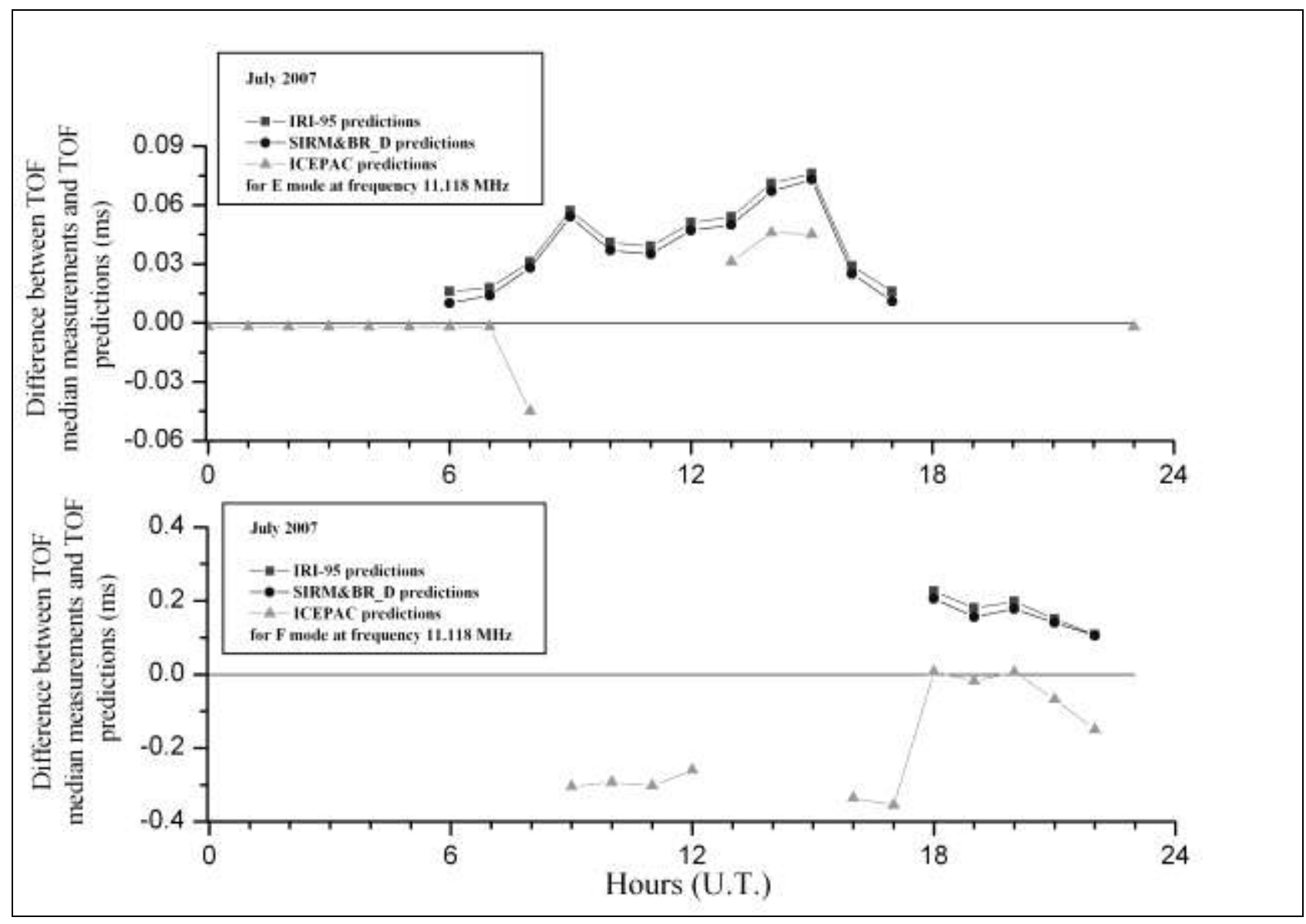

Figure 6 


\begin{tabular}{|c|c|c|c|}
\hline $1 \mathrm{E}$ propagation mode & $\begin{array}{l}\text { Winter months } \\
\text { r.m.s min }- \text { r.m.s. } \max \\
(\mathrm{ms})\end{array}$ & $\begin{array}{l}\text { Equinoctial months } \\
\text { r.m.s min }- \text { r.m.s. max } \\
\text { (ms) }\end{array}$ & $\begin{array}{l}\text { Summer months } \\
\text { r.m.s min }- \text { r.m.s. } \max \\
(\mathrm{ms})\end{array}$ \\
\hline IRI-95 & $0.038-0.099$ & $0.044-0.085$ & $0.013-0.093$ \\
\hline SIRM\&BR_D & $0.045-0.099$ & $0.043-0.084$ & $0.008-0.072$ \\
\hline ICEPAC & $0.002-0.048$ & $0.022-0.062$ & $0.002-0.082$ \\
\hline
\end{tabular}

Table 1: The minimum and maximum value of the r.m.s. error are shown taking into account all the frequencies which arrive at the receiver following the $1 \mathrm{E}$ on great circle propagation modes. 


\begin{tabular}{|c|c|c|c|}
\hline $1 \mathrm{~F}$ propagation mode & $\begin{array}{l}\text { Winter months } \\
\text { r.m.s min }- \text { r.m.s. } \max \\
\text { (ms) }\end{array}$ & $\begin{array}{l}\text { Equinoctial months } \\
\text { r.m.s min }- \text { r.m.s. } \max \\
\text { (ms) }\end{array}$ & $\begin{array}{l}\text { Summer months } \\
\text { r.m.s min }- \text { r.m.s. } \max \\
(\mathrm{ms})\end{array}$ \\
\hline IRI-95 & $0.071-0.325$ & $0.072-0.393$ & $0.111-0.572$ \\
\hline SIRM\&BR_D & $0.097-0.139$ & $0.070-0.254$ & $0.108-0.340$ \\
\hline ICEPAC & $0.025-0.325$ & $0.093-0.396$ & $0.095-0.432$ \\
\hline
\end{tabular}

Table 2: The minimum and maximum value of the r.m.s. error are shown taking into account all the frequencies which arrive at the receiver following the $1 \mathrm{~F}$ on great circle propagation modes. 
Winter months Equinoctial months Summer months

$\mathrm{DP}_{\text {min }}-\mathrm{DP}_{\text {max }} \quad \mathrm{DP}_{\text {min }}-\mathrm{DP}_{\text {max }} \quad \mathrm{DP}_{\text {min }}-\mathrm{DP}_{\text {max }}$

\begin{tabular}{cccc} 
& $(\mathrm{km})$ & $(\mathrm{km})$ & $(\mathrm{km})$ \\
\hline IRI-95 & $4.3-28.0$ & $10.5-24.9$ & $3.8-23.1$ \\
\hline SIRM\&BR_D & $8.1-27.9$ & $9.6-24.6$ & $2.4-19.6$ \\
\hline ICEPAC & $0.1-5.27$ & $0.5-15.6$ & $0.6-16.6$
\end{tabular}

Table 3: minimum and maximum DP values for different seasons and prediction methods for $1 \mathrm{E}$ propagation mode 
Winter months Equinoctial months Summer months

$\mathrm{DP}_{\min }-\mathrm{DP}_{\max } \quad \mathrm{DP}_{\text {min }}-\mathrm{DP}_{\max } \quad \mathrm{DP}_{\text {min }}-\mathrm{DP}_{\text {max }}$

$(\mathrm{km}) \quad(\mathrm{km}) \quad(\mathrm{km})$

\begin{tabular}{cccc}
\hline IRI-95 & $20.1-68.9$ & $16.4-85.5$ & $32.1-171.0$ \\
\hline SIRM\&BR_D & $27.7-38.5$ & $24.6-63.6$ & $32.2-88.6$ \\
\hline ICEPAC & $2.3-49.5$ & $22.9-85.2$ & $4.6-127.9$
\end{tabular}

Table 4: minimum and maximum DP values for different seasons and prediction methods for $1 \mathrm{~F}$ propagation mode 